\title{
Higher-Order Continuum Equation Based Heat Conduction Law
}

\author{
Amit Agrawal $^{1}$
}

Received: 13 May 2016/Accepted: 22 June 2016/Published online: 15 July 2016

(C) Indian National Academy of Engineering 2016

\begin{abstract}
The Fourier law has the unphysical consequence of infinite speed of heat conduction. The Cattaneo modification to the heat conduction equation removes this deficiency. The modified equation however suffers from two main defects: the speed of propagation cannot be determined theoretically, and the equation can lead to results which violate the second law of thermodynamics. In this paper, we derive the Cattaneo equation starting from Grads 13-moment equations. Further, we derive a thermodynamically consistent conduction model from the regularized moment equations. The deficits in the existing models are therefore satisfactorily addressed in this work.
\end{abstract}

Keywords Non-Fourier heat conduction - Nanoscale heat transfer - Cattaneo equation · 13-moment equations . Relaxation time . Thermodynamically consistent heat conduction model

\section{Introduction}

The Fourier law suggests a linear relation between heat flux and temperature gradient. This law is widely used and has been shown to yield satisfactory result in numerous cases. The basic problem with this law, however, is that it assumes that the heat flux is instantaneously set up (or vanishes) upon application (or removal) of a temperature gradient. Since no process is instantaneous, a correction is required to this law. Such a correction becomes especially pertinent in problems

Amit Agrawal

amit.agrawal@iitb.ac.in

1 Indian Institute of Technology Bombay, Powai, Mumbai 400076, India involving small length (Yang et al. 2005; Shiomi and Maruyama 2006) or time scale (Tzou and Chiu 2001; Mishra and Sahai 2012) and for temperatures close to absolute zero (Ozisik and Vick 1984). Chester (1963) mentions that there is a critical frequency beyond which non-Fourier effects become appreciable. Another application of nonFourier conduction is in crystals involving exothermal catalytic reaction with temperature rise happening very rapidly (Ozisik and Vick 1984). According to Cao and Guo (2007), the Fourier's law can be viewed as the balance between phonon gas pressure gradient (driving force) and flow resistance in the phonon gas. However when the heat flux density becomes large, the inertial force on the phonon gas cannot be neglected, and non-Fourier heat conduction effects need to be accounted for.

The most commonly applied modification of the Fourier law is the Cattaneo equation, proposed independently by Cattaneo (1958) and Vernotte (1958) at around the same time:

$\tau \frac{\partial q_{i}}{\partial t}+q_{i}=-k \frac{\partial T}{\partial x_{i}}$.

In the above equation, $q_{i}$ is heat flux vector, $T$ is temperature, $k$ is thermal conductivity, $\tau$ is relaxation time, $x_{i}$ is spatial coordinate, and $t$ is time. Cattaneo's modification is however rather arbitrary (Chester 1963), and leads to the following two problems. First, the value of the relaxation time needs to be supplied; obtaining its value theoretically appears difficult. Some studies have suggested equating the relaxation time to diffusity $(\alpha)$ and thermal wave speed $(c)$ as $\tau=\alpha / c^{2}$. Chester (1963) suggested an expression for relaxation time in terms of phonon velocity. However, the value of thermal wave speed or phonon velocity in different media is not known. Second, the nature of the above equation being hyperbolic in nature leads to wave-like 
motion of heat. Constructive interference of the waves can lead to the value of temperature being more than the physical limit. Several examples of violations of the second law of thermodynamics are available in the literature (Taitel 1972; Rubin 1992; Ali and Zhang 2005).

The above studies therefore suggest that although the Cattaneo's equation is a useful modification of the Fourier law, it is incomplete (Roetzel et al. 2003).Tzou (1995) introduced the following dual-lag equation to allow for the lagging behaviour:

$q_{i}\left(\bar{x}, t+\tau_{q}\right)=-k \frac{\partial T}{\partial x_{i}}\left(\bar{x}, t+\tau_{T}\right)$.

The above equation upon expanding in Taylor series, and neglecting second and higher order terms, yields:

$q_{i}+\tau_{q} \frac{\partial q_{i}}{\partial t}=-k\left(\frac{\partial T}{\partial x_{i}}+\tau_{T} \frac{\partial}{\partial t} \frac{\partial T}{\partial x_{i}}\right)$.

Notice the introduction of two time constants $\left(\tau_{q}, \tau_{T}\right)$ in the above equation, which makes its application even more difficult. However, the other issues with the Cattaneo model (equation not having a fundamental basis, and wavelike solution leading to unphysical results) remain.

Recognizing the above issues, attempts have been made to derive non-Fourier models from alternate ways. Chen (2001) proposed a diffusive-ballistic model, where the information coming from the wall (the ballistic part) is suitably added to the diffusive component. This approach leads to a Cattaneo-like equation, with a ballistic term added to the equation. The proposed equation was tested for heat conduction in a thin film and the obtained solution was compared against the solution obtained from the Boltzmann equation. The obtained solution from the ballistic-diffusive model for heat flux compares better to the Boltzmann equation, than that obtained from the Fourier and Cattaneo's equations. However, the solution for non-dimensional temperature shows discrepency (Chen 2002). The application of the ballistic-diffusive equation appears difficult; moreover, the proposed equation has not been tested for complex problems. An alternative recent attempt to derive the Cattaneo's equation has been made by Maassen and Lundstrom (2015a, b) using the McKelvey-Shockley flux model. In this approach, fluxes coming from positive and negatives sides are treated separately. Ali and Zhang (2005) derived the relativistic form of heat conduction equation. Further comparison with these equations is given later.

In this work, we show that the derivation of Cattaneo's equation is possible starting from higher-order continuum transport equations. The obtained equation contains an additional term that solves the problem of temperature becoming unphysical. The value of relaxation time for dilute gas modeled as Maxwellian molecule is found to be $3 \mu /(2 p)$, where $\mu$ is the viscosity and $p$ is pressure.

\section{Derivation and Results}

In this paper, we use the 13-moments equations. The 13moments equations were first derived by Grad (1949) starting from the Boltzmann's equation. Here, density $\rho$, velocity vector $v_{i}$, temperature $T$, heat flux vector $q_{i}$, and pressure tensor $p_{i j}$ are all treated as unknowns (13 unknowns in total) and appropriate equations are written down for these variables. Of late, there has been some success in solving higher-order continnum equations analytically for fluid flow problems (Singh and Agrawal 2014; Singh et al. 2014a, b).

The equations for the above mentioned variables are (Taheri et al. 2009):

$\frac{\partial \rho}{\partial t}+\frac{\partial \rho v_{k}}{\partial x_{k}}=0$

$\rho \frac{\partial v_{i}}{\partial t}+\rho v_{k} \frac{\partial v_{i}}{\partial x_{k}}+\frac{\partial p}{\partial x_{i}}+\frac{\partial \sigma_{i k}}{\partial x_{k}}=0$

$\frac{3}{2} \rho \frac{\partial R T}{\partial t}+\frac{3}{2} \rho v_{k} \frac{\partial R T}{\partial x_{k}}+\frac{\partial q_{k}}{\partial x_{k}}+p \frac{\partial v_{k}}{\partial x_{k}}+\sigma_{i j} \frac{\partial v_{i}}{\partial x_{j}}=0$

$\frac{D \sigma_{i j}}{D t}+\sigma_{i j} \frac{\partial v_{k}}{\partial x_{k}}+\frac{4}{5} \frac{\partial q_{<i}}{\partial x_{j}>}+\frac{\partial \rho_{<i j k>}}{\partial x_{k}}+2 p \frac{\partial v_{<i}}{\partial x_{j}>}$

$$
+2 \sigma_{k<i} \frac{\partial v_{j}>}{\partial x_{k}}=-\frac{p \sigma_{i j}}{\mu}
$$

$$
\begin{aligned}
\frac{D q_{i}}{D t} & +\frac{5}{2} p \frac{\partial R T}{\partial x_{i}}-\sigma_{i k} R T \frac{\partial \ln \rho}{\partial x_{k}}-\frac{\sigma_{i j}}{\rho} \frac{\partial \sigma_{j k}}{\partial x_{k}}+\frac{5}{2} \sigma_{i k} \frac{\partial R T}{\partial x_{k}} \\
& +R T \frac{\partial \sigma_{i k}}{\partial x_{k}}+\frac{7}{5} q_{k} \frac{\partial v_{i}}{\partial x_{k}}+\frac{7}{5} q_{i} \frac{\partial v_{k}}{\partial x_{k}}+\frac{2}{5} q_{k} \frac{\partial v_{k}}{\partial x_{i}}+\frac{1}{2} \frac{\partial \rho_{r r<i k>}}{\partial x_{k}} \\
& +\frac{1}{6} \frac{\partial \rho_{r r s s}}{\partial x_{i}}+\rho_{<i j k}>\frac{\partial v_{j}}{\partial x_{k}}=-\frac{2}{3} \frac{p q_{i}}{\mu}
\end{aligned}
$$

where angular brackets denote trace-free symmetric tensor, and $R$ denotes gas constant. Further, $\rho_{r r s s}, \rho_{<i j k>}, \rho_{r r<i j>}$ are higher-order moments generated in the process of the derivation. These terms are assumed to be zero in the Grad's formulation. The closure equations for these terms in the R13 formulation are (Taheri et al. 2009):

$$
\begin{aligned}
\rho_{r r s s}= & -\frac{\sigma_{i j} \sigma_{i j}}{\rho}-12 \frac{\mu}{p}\left(R T \frac{\partial q_{k}}{\partial x_{k}}+\frac{5}{2} q_{k} \frac{\partial R T}{\partial x_{k}}-R T q_{k} \frac{\partial \ln \rho}{\partial x_{k}}\right. \\
+ & \left.R T \sigma_{k l} \frac{\partial v_{k}}{\partial x_{l}}\right) \\
\rho_{r r<i j>}= & -\frac{4}{7} \frac{\sigma_{k<i} \sigma_{j>k}}{\rho}-\frac{24}{5} \frac{\mu}{p}\left(R T \frac{\partial q_{<i}}{\partial x_{j}>}+q_{<i} \frac{\partial R T}{\partial x_{j}>}\right. \\
& \left.-R T q_{<i} \frac{\partial \ln \rho}{\partial x_{j}>}+\frac{10}{7} R T \sigma_{k<i} S_{j>k}\right) \\
\rho_{<i j k>}= & -2 \frac{\mu}{p}\left(R T \frac{\partial \sigma_{<i j}}{\partial x_{k}>}-R T \sigma_{<i j} \frac{\partial \ln \rho}{\partial x_{k}>}+\frac{4}{5} q_{<i} \frac{\partial v_{j}}{\partial x_{k}>}\right)
\end{aligned}
$$


where $S_{j k}=\partial v_{<j} / \partial x_{k}>$.

Setting $v_{j}=\sigma_{i j}=0$ in the above equations, and utilizing the equation of state $(p=\rho R T)$, helps reduce the above equations to

$\frac{\partial \rho}{\partial t}=0$

$\frac{\partial p}{\partial x_{k}}=0$

$\frac{3}{2} \rho \frac{\partial R T}{\partial t}+\frac{\partial q_{k}}{\partial x_{k}}=0$

$\frac{4}{5} \frac{\partial q_{<i}}{\partial x_{j}>}=0$

$\frac{\partial q_{i}}{\partial t}+\frac{5}{2} p \frac{\partial R T}{\partial x_{i}}+\frac{1}{2} \frac{\partial \rho_{r r<i k}>}{\partial x_{k}}+\frac{1}{6} \frac{\partial \rho_{r r s s}}{\partial x_{i}}=-\frac{2}{3} \frac{p q_{i}}{\mu}$

along with the following closure relations:

$$
\begin{aligned}
& \rho_{r r s s}=-12 \frac{\mu}{p}\left(R T \frac{\partial q_{k}}{\partial x_{k}}+\frac{5}{2} q_{k} \frac{\partial R T}{\partial x_{k}}-R T q_{k} \frac{\partial \ln \rho}{\partial x_{k}}\right) \\
& \rho_{r r<i j>}=-\frac{24}{5} \frac{\mu}{p}\left(q<i \frac{\partial R T}{\partial x_{j}>}-R T q_{<i} \frac{\partial \ln \rho}{\partial x_{j}>}\right) \\
& \rho_{<i j k>}=0 .
\end{aligned}
$$

Simple algebric manipulation of Eq. 16 yields:

$\frac{3}{2} \frac{\mu}{p} \frac{\partial q_{i}}{\partial t}+q_{i}=-k \frac{\partial T}{\partial x_{i}}-\frac{3}{4} \frac{\mu}{p} \frac{\partial \rho_{r r<i k}>}{\partial x_{k}}-\frac{\mu}{4 p} \frac{\partial \rho_{r r s s}}{\partial x_{i}}$.

The relation $k=(15 / 4) \mu R$ has been utilized in writing the above equation. Notice that Eq. 20 is same as Eq. 1 albeit with two additional terms. The relaxation term can be recognized as $(3 / 2) \mu / p$. The value of relaxation time for air at standard condition is approximately $2.7 \times 10^{-10} \mathrm{~s}$. A similar order of relaxation time has been provided by Tzou and Chiu (2001). As mentioned above, the additional terms are zero in Grad's formulation, whereas the additional terms are given by Eqs. 17 and 18 in R13 formulation. An improved set of 13-moment equations have recently been derived by Singh and Agrawal (2016).

\section{Discussion}

The reason for invoking the 13-moments equations here is justified because in these equations no apriori relation is assumed between the heat flux vector and temperature gradients. This allows determination of the relation between them, as shown above. Note that the 13-moment equations have been derived from Boltzmann equation for dilute monatomic gases; and molecules have been assumed as Maxwellian in the solution of the collision term. The derivation of the Cattaneo's equation requires no further assumption and is therefore regarded as having being derived from first-principles. Of course, the derivation is strictly valid for dilute, monatomic, Maxwellian gases, and its further extension to liquid is difficult to prove. The present approach is therefore somewhat akin to that utilized for derivation of Stoke's hypothesis, where the relation between the shear and dilation viscosities is first derived for monatomic gases and subsequently the obtained relation is applied to all fluids.

Taitel (1972) and Rubin (1992) (among several others) argued that the Cattaneo's model can lead to flow of heat from cold region to hot region, which would violate the second law of thermodynamics. Ali and Zhang (2005) argued that the time derivative of heat flux allows leakage of heat along the time direction. This unaccounted leakage of heat can lead to violation of the laws of thermodynamics. Rubin (1992) proposed alternative constitutive equations to overcome this deficiency. The internal energy, Helmholtz free energy, and entropy relation were modified by Rubin to include dependence on temperature rate. Shnaid (2003) claimed to have derived the hyperbolic heat conduction equation within the tenet of classical thermodynamics. Berkovsky and Bashtovoi (1977) analyzed the issue from kinetic theory point of view. They suggested generalizing the collision term in the Boltzmann equation to overcome this issue. Barletta and Zanchini (1997) recognized that if the temporal gradient of heat flux varies more rapidly than the value of heat flux divided by relaxation time, then the entropy production term becomes negative. Such a situation was found to arise in the example cases considered by them. Their contention however was that the temperature cannot be defined since the local thermodynamic equilibrium assumption is violated in the problem.

As stated earlier, Eq. 20 is similar to Cattaneo equation, with two addition terms on the right hand side. The equation reduces exactly to Cattaneo equation in Grad's formulation. The approach of Maassen and Lundstrom (2015a, b) also yields the Cattaneo equation without any additional terms. The presence of additional terms is noted in the approach of Chen (2001), although the above term is very different from that obtained by Chen. Notice that the additional terms have $(\mu / p)^{2}$ as their coefficient, indicating that these are higher-order terms. The Fourier's law, which is correct to the first order, understandably does not have these higher-order terms. A non-dimensional analysis of the equation (not presented here) shows that the higherorder terms become important at high Knudsen numbers (i.e., when the characteristic length scale becomes comparable to the mean free path of the gas), which is relevant in micro- and nano-scale thermal problems. A similar non- 
dimensional analysis shows that the transient term is important for time comparable to the relaxation time $\tau$. That is, a very short interval after application of temperature gradient, or thermal problems involving temperature variation at ultra-high frequency.

The H-theorem (i.e., entropy consistency) of the R13 equation has already been proved Struchtrup and Torrilhon (2007). Since Eq. 20 has been derived from the R13 equation without any additional assumption, the proposed equation is also entropy consistent. Moreover, the validity of the R13 equations has been checked against direct simulation Monte Carlo data, for Couette and Poiseuille flows (Taheri et al. 2009), which lends further credence to the equation proposed herein. The useful of Catteneo equation has been shown by its application to various problems (Shiomi and Maruyama 2006; Tzou and Chiu 2001; Mishra and Sahai 2012; Xu et al. 2008; Antaki 2005). The purpose of this work is not to validate the model proposed in Eq. 20, rather to show the connection between the Grad and Cattaneo's equations, which is not available earlier. The validity of the proposed model will depend on the accuracy of the closure relations (Eqs. 17, 18). Derivation of better closure models such as done by Singh and Agrawal (2016) will improve the non-Fourier model for analyzing problems involving small length or time scale; and for temperatures close to absolute zero.

\section{Concluding Remarks}

In this work, we employ higher-order continuum transport equations to derive a non-Fourier heat conduction relation. The advantage of employing this approach is that the heat flux vector and temperature gradients are treated as independent variables, and suitable relationship between these variables is derived from the Boltzmann's equation using the Grad's moment approach.

We are able to show that the Grad's 13-moment equations lead exactly to the Cattaneo model. Our derivation therefore provides a first-princple based derivation of the Cattaneo equation. Further, we show that the regularized 13-moment equation leads to Cattaneo-like equation with two additional terms. We believe that these additional terms makes the proposed equation (Eq. 20) thermodynamically consistent. In addition our study provides a way to relate the relaxation time to measurable macroscopic parameters. Note that other rather difficult to measure parameters (such as thermal wave speed in different gaseous media and different temperatures) can be further determined from the present approach.

The work however does not lend support to the dualrelaxation model available in the literature. The proposed equation (Eq. 20) needs to be thoroughally tested (especially in terms of thermodynamic consistency of the results) to establish its superiority with respect to the available models.

Acknowledgments This work was done as part of the Department of Atomic Energy (DAE-SRC) Outstanding Investigator Award project.

\section{References}

Ali YM, Zhang LC (2005) Relativistic heat conduction. Int J Heat Mass Transfer 48:2397-2406

Antaki PJ (2005) New interpretation of non-fourier heat conduction in processed meat. J Heat Transfer 123:189-193

Barletta A, Zanchini E (1997) Hyperbolic heat conduction and local equilibrium: a second law analysis. Int $\mathbf{J}$ Heat Mass Transfer 40:1007-1016

Berkovsky BM, Bashtovoi VG (1977) The finite velocity of heat propagation from the viewpoint of the kinetic theory. Int $\mathrm{J}$ Heat Mass Transfer 20:621-626

Cao BY, Guo ZY (2007) Equation of motion of a phonon gas and non-Fourier heat conduction. J Appl Phys 102:053503

Cattaneo C (1958) A form of heat conduction equation which eliminates the paradox of instantaneous propagation. Compte Rendus 247:431-433

Chen G (2001) Ballistic-diffusive heat conduction equations. Phys Rev Lett 86:2230-2297

Chen G (2002) Ballistic-diffusive equations for transient heat conduction from nano to macroscales. $J$ Heat Transfer 124:320-328

Chester M (1963) Second sound in solids. Phys Rev 131:2013-2015

Grad H (1949) On the kinetic theory of rarefied gases. Comm Pure Appl Math 2:331

Maassen J, Lundstrom M (2015a) Steady-state heat transport: ballistic-to- diffusive with Fouriers law. J Appl Phys 117:035104

Maassen J, Lundstrom M (2015b) A simple Boltzmann transport equation for ballistic to diffusive transient heat transport. J Appl Phys 117:135102

Mishra SC, Sahai H (2012) Analyses of non-Fourier heat conduction in 1-D cylindrical and spherical geometry-an application of the lattice Boltzmann method. Int $\mathrm{J}$ Heat Mass Transfer 55:7015-7023

Ozisik MN, Vick B (1984) Propagation and reflection of thermal waves in a finite medium. Int $\mathbf{J}$ Heat Mass Transfer 27:1845-1854

Roetzel W, Putra N, Das SK (2003) Experiment and analysis for nonFourier conduction in materials with non-homogeneous inner structure. Int J Thermal Sci 42:541-552

Rubin MB (1992) Hyperbolic heat conduction and the second law. Int J Eng Sci 30:1665-1676

Shiomi J, Maruyama S (2006) Non-Fourier heat conduction in a single-walled carbon nanotube: classical molecular dynamics simulations. Phys Rev B 73:205420

Shnaid I (2003) Thermodynamically consistent description of heat conduction with finite speed of heat propagation. Int J Heat Mass Transfer 46:3853-3863

Singh N, Agrawal A (2014) The Burnett equations in cylindrical coordinates and their solution for flow in a microtube. J Fluid Mech 751:121-141

Singh N, Agrawal A (2016) Onsager's-principle-consistent 13-moment transport equations. Phys Rev E 93:063111

Singh N, Dongari N, Agrawal A (2014) Analytical solution of plane Poiseuille flow within Burnett hydrodynamics. Microfluidics Nanofluidics 16:403-412 
Singh N, Gavasane A, Agrawal A (2014) Analytical solution of plane Couette flow in the transition regime and comparison with direct Simulation Monte Carlo data. Comput Fluids 97:177-187

Struchtrup H, Torrilhon M (2007) H theorem, regularization, and boundary conditions for linearized 13 moment equations. Phys Rev Lett 99:014502

Taheri P, Torrilhon M, Struchtrup H (2009) Couette and Poiseuille microflows: Analytical solutions for regularized 13-moment equations. Phys Fluids 21:017102

Taitel Y (1972) On the parabolic, hyperbolic and discrete formulation of the heat conduction equation. Int $\mathrm{J}$ Heat Mass Transfer 15:369-371

Tzou DY (1995) A unified field approach for heat conduction from macro- to micro-scales. J Heat Transfer 117:8-16
Tzou DY, Chiu KS (2001) Temperature-dependent thermal lagging in ultrafast laser heating. Int J Heat Mass Transfer 44:1725-1734

Vernotte P (1958) Les paradoxes de la theorie continue de l'equation de la chaleur. Compte Rendus 246:3154-3155

$\mathrm{Xu} \mathrm{F}$, Seffen KA, Lu TJ (2008) Non-Fourier analysis of skin biothermomechanics. Int J Heat Mass Transfer 51:2237-2259

Yang HQ (1991) Non-Fourier effect on heat conduction during welding. Int J Heat Mass Transfer 34:2921-2924

Yang R, Chen G, Laroche M, Taur Y (2005) Simulation of nanoscale multidimensional transient heat conduction problems using ballistic-diffusive equations and phonon boltzmann equation. J Heat Transfer 127:298-306 